\title{
Narrow Lines and Continuum Emission in Seyfert 2 Galaxies
}

\author{
M. Polletta, L. Bassani, E. Caroli, G. Malaguti, A. Malizia, and G. G. C. \\ Palumbo \\ ITeSRE/CNR, via Gobetti 101, 40129 Bologna, Italy
}

\begin{abstract}
A statistical analysis of the relationship between the continuum and the narrow emission lines of a sample of Seyfert 2 galaxies strongly suggests the presence of a starburst emitting at all wavelengths from radio to soft X-rays as well as in the narrow lines.
\end{abstract}

\section{Introduction}

The unified model for AGNs assumes that in Seyfert 2 galaxies the primary radiation coming from the nuclear region and escaping from the absorbing torus is still able to photoionize the narrow-line region. The present work is an attempt to test this model by analyzing the relationship between narrow-line emission and continuum luminosities. A sample containing all Seyfert 2 galaxies observed in the hard X-ray band (2-10 keV) has been selected (Polletta et al. 1996) and is considered here. The sample contains 150 Seyfert galaxies classified as type $1.8,1.9$, or 2 , or as NELGs. For each source, continuum data in all energy bands (radio at $\lambda=6 \mathrm{~cm}$; infrared at $\lambda=100,60,25,12$, and $3.5 \mu \mathrm{m}$; ultraviolet at $\lambda=1450 \AA$, soft X-ray at $E=0.1-3 \mathrm{keV}$, and hard X-ray at $E=2-10 \mathrm{keV}$ ) and the $\mathrm{H} \beta$ and [O $\mathrm{III}$ ] emission lines are available. The hard X-ray luminosity which characterizes this sample can be used as an indicator of the energy emitted from the most compact region of Seyfert 2 galaxies. Such radiation is free from emission coming from the extranuclear and stellar components.

\section{Data Analysis and Results}

In the statistical analysis both Pearson's and Spearman's tests have been applied. The best results obtained have been reported here. The correlations obtained (Pearson's $r(\mathrm{H} \beta-[\mathrm{O}$ III $])=0.86$ with a confidence level $C L>99.99 \%$ ) indicate that the same physical mechanism produces the emission in the narrow $\mathrm{H} \beta$ and [O $\mathrm{III}]$ lines, and hard X-rays and lines appear to be correlated, although weakly (Pearson's $r(\mathrm{H} \beta-\mathrm{HX})=0.64, C L>99.8 \%$ and $r([\mathrm{O} \mathrm{HI}]-\mathrm{HX})=0.59$, $C L \approx 99.4 \%$ ). Photoionization therefore seems to be still valid.

The line intensities seem also to have a common component with the radio and the infrared sources. The small number of ultraviolet data in the present sample does not allow us to establish whether there is a common component between lines and the ultraviolet source. The analysis of the relationship between the narrow emission lines $\mathrm{H} \beta$ and [O III] and the continuum (Spearman's 
$r(\mathrm{H} \beta$-Radio $)=0.61$, Spearman's $r([\mathrm{O}$ III $]$-Radio $)=0.60$, Pearson's $r(\mathrm{H} \beta$-IR $)=$ 0.71 , Pearson's $r([\mathrm{O} I \mathrm{III}]-\mathrm{IR})=0.60$, and Spearman's $r(\mathrm{H} \beta-\mathrm{UV})=0.72$, all with $C L>99.99 \%$ ) indicates that the emission in these lines depends both on the nuclear source (probably due to photoionization) and on the continuum emitted at lower energies.

The $[\mathrm{O} \mathrm{III}] / \mathrm{H} \beta$ flux ratio is a measure of the ionization state of the emitting region. For Seyfert 2 galaxies, $[\mathrm{O} \mathrm{II}] / \mathrm{H} \beta$ is expected to be $>3$ (Shuder \& Osterbrock 1981). This criterion can be understood qualitatively in terms of the differences between a stellar-type photoionizing continuum $([\mathrm{O} \mathrm{III}] / \mathrm{H} \beta<3)$ and a power-law type, extending to much higher energies. Nineteen sources $(\sim 25 \%)$ in the sample show $[\mathrm{OIII}] / \mathrm{H} \beta<3$. For these sources, a stellar-type (starburst) photoionizing continuum produces the emission in the $\mathrm{H} \beta$ and [O III] lines.

The emission in the infrared and radio is not correlated with the hard $\mathrm{X}$-ray luminosity, but is correlated with the soft X-ray luminosity (Pearson's $r(\mathrm{SX}-\mathrm{Radio})=0.69$ with $C L \approx 99.8 \%$ and $r(\mathrm{SX}-\mathrm{IR})=0.64$ with $C L>$ $99.99 \%)$. Furthermore, the infrared continuum shows a significant correlation rank with the radio and ultraviolet continua (Spearman's $r$ (IR-Radio) $=0.68$ with $C L>99.99 \%$ and Pearson's $r(\mathrm{IR}-\mathrm{UV})=0.69$ with $C L>99.99 \%)$. These results can be explained by the presence of a continuum component emitting from the radio to the soft $\mathrm{X}$-ray band that is not connected with the nuclear source. Such a component could be attributed to a starburst.

\section{Conclusions}

A statistical analysis applied to radio, infrared, ultraviolet, soft X-ray continuum and emission-line luminosities of a selected sample of hard X-ray emitting Seyfert 2 galaxies indicates that in these bands a starburst component may be present. This is a well-known fact for a few Seyfert 2 galaxies which show circumnuclear starburst activity (Storchi-Bergmann et al. 1992). However the same does not apply to Seyfert 1 galaxies. This seems to contradict the unified model, unless the starburst component is present in both Seyfert types, but is harder to recognize in Seyfert 1 galaxies because the nuclear emission dominates over other components, while in Seyfert 2 galaxies the starburst activity is observable because the nuclear emission is heavily absorbed by the torus and therefore is not dominant. However the results reported here can also be explained assuming an evolutionary scenario (Maiolino et al. 1995), which is in contradiction to the unified model.

Acknowledgments. Financial support by MURST, CNR and ASI is gratefully acknowledged.

\section{References}

Maiolino, R., Ruiz, M., Rieke, G.H., \& Keller, L.D. 1995, ApJ, 446, 561.

Polletta, M., Bassani, L., Malaguti, G., Palumbo, G.G.C., \& Caroli, E. 1996, ApJS, in press.

Shuder, J. M., \& Osterbrock, D.E. 1981, ApJ, 250, 55.

Storchi-Bergmann, T., Mulchaey, J.S., \& Wilson, A.S. 1992, ApJ, 395, L73. 